\title{
Editorial
}

\section{Application of Radiation Cross-linking in Automotive Industry}

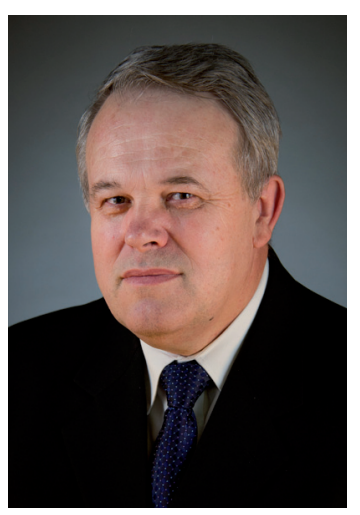

prof. Ing. František Greškovič, PhD.

Technical University of Košice, Faculty of Mechanical Engineering, Department of Automotive Production

František Greškovič, prof., Ing., CSc., (born in 1956) is a professor at the Technical University of Košice, Faculty of Mechanical Engineering, Institute of Technologies and Management. He graduated from the Faculty of Mechanical engineering in 1980. His research areas are processing and simulation of injection molding, as well as die design in CAD/CAM systems and 3D modelling. He is an author/co-author of 1 monography, 3 textbooks and more than 170 publications in journals and conference proceedings in Slovakia and abroad. Eight papers were registered in Web of Science and SCOPUS. He is co-author of nine patents. He has been involved in several grant projects and research tasks.

The cross-linking is the most important reaction of polymers that significantly changes product properties. In the cross-linking, the molecules are joined to create a functional net. The radiation in cross-linking causes change of the thermoplastic into the thermoset. Increasing density of cross-linking increases rigidity and hardness, electric resistance and resistance to solvents; and decreases the degree of bulking. The cross-linking is carried out by peroxides, silanes, ionizing radiation (for example the radiation dose in PE is within the range $1-30 \mathrm{Mrad}$ ) and by vulcanization of sulphur (in caoutchouc).

Higher temperatures are usually used (polymer is melted) in cross-linking by peroxides (dibenzoylperoxide, butylperoxide). In the first step, peroxide (ROOR) is decomposed by heat into free radicals $R O$ which further react with polymer string. In the recombination of polymer radicals, there occurs joining of string through the C-C bond.

The disadvantages of cross-linking is its low efficiency (side reactions of peroxides and free radicals) and necessity of using the big amount of relatively expensive peroxides as well as mixing the compound of polymers with peroxides and stabilizers in a special equipment (in PE Engel process).

Peroxide is used to create a primary radical. Molecules of silane are then inoculated with primary radicals. Then Si-O-Si bridges are created. For the optimal speed of reaction, elevated temperature is used (in PE from $80^{\circ} \mathrm{C}$ to $90^{\circ} \mathrm{C}$ ) with the presence of catalyst. The advantage of cross-linking by silanes is in using conventional machines, and in addition, wider temperature range is available in comparison to the cross-linking by peroxides. The problem is the byproducts occurrence (methanol and water) during reaction.

The cross-linking includes the basic assumption, that the materials during ionising radiation are mainly cross-linked and do not degrade. It is a physical method and chemical ingredients are 


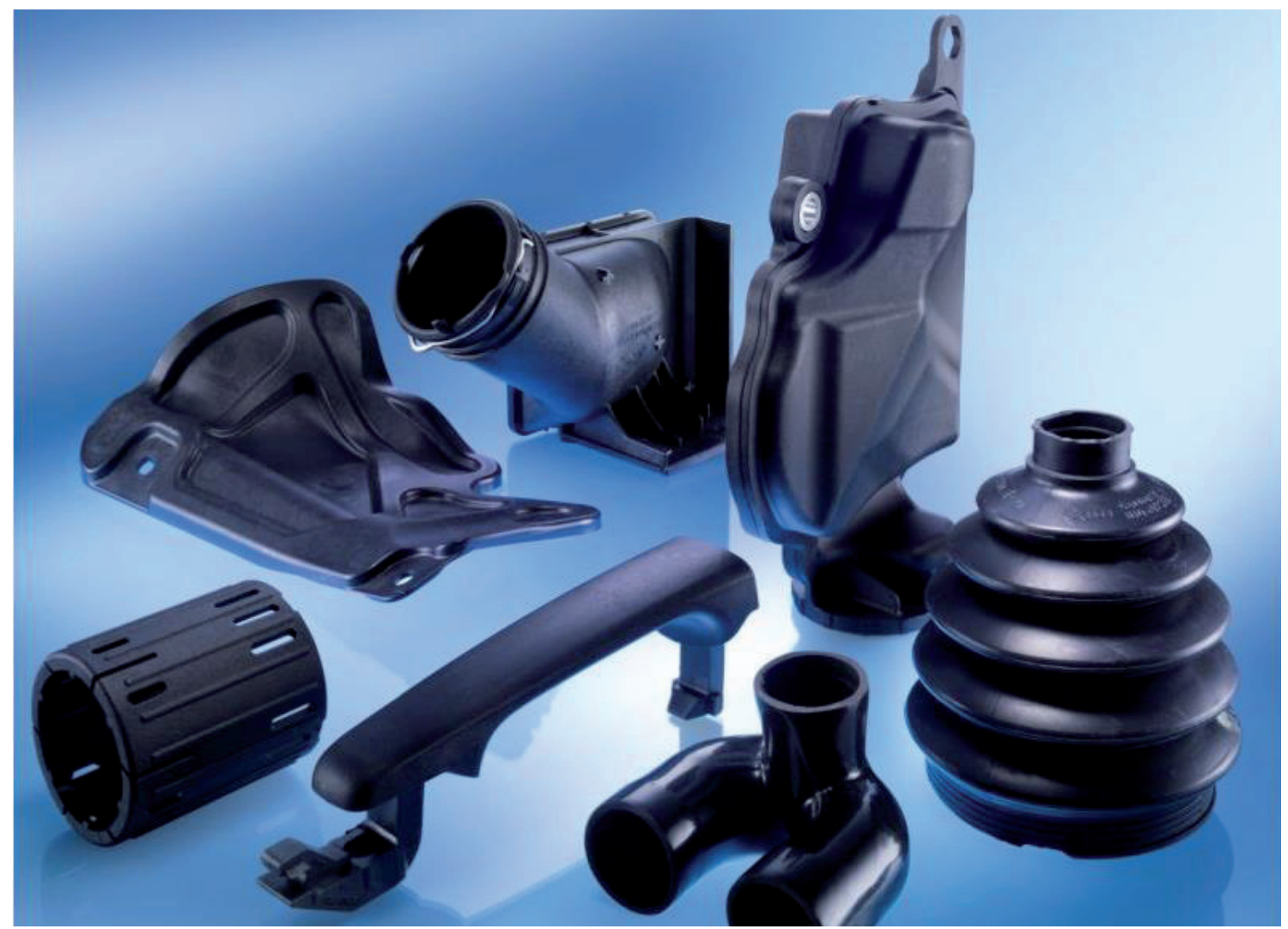

not needed (for example in PP a cross-linking reagent TAIC is used). The main condition is the presence of three or more functional monomers. The interaction of radiation and polymers causes the creation of polymer radicals (decomposition of bonds $(-H)$ ), which create nets by recombination in strings. A net is created by bonding of two free radicals between adjacent strings with creation of a C-C bond. The cross-linking is performed mostly by beta or gamma radiation. Because of radiation, thermoplastics are transformed into the materials with the properties of elastomers in certain temperatures. The elastomers or partially non cross-linked systems have a sufficient number of crosslinked areas due to radiation. It is not necessary to use the elevated temperature during the radiation crosslinking process. The cross-linking is always used after processing.

Electrons can be accelerated artificially in so-called electron accelerators, for example transformation type Betatron or electrostatic cathodic.

The properties of a composite filled with an element filler depend on the physical properties of components (matrix, filler). The coherence of the matrix with the filler has a significant influence on the transmission of stresses on reinforcement as well as on the mechanical properties.

The radiation technology of beta or gamma rays is utilized to achieve joining of the matrix structure with the reinforcement to increase the material strength.

The evaluation of composite structure is usually based on the fracture area after tensile test. It is realized by REM RE-Detector with given magnification. The material filled with glass short fibres PA6 GF30 is shown in figure above. Its structure is irradiated by dose of 100 kGy.

Using of regrinds from irradiated material in a repeated process of injection prevents the irradiated material from melting, which can affect the properties, as well as the appearance of the component.

The method of the injection cycle reduction or cooling time can be reduced in some cases by about $40 \%$. 